TITLE:

\title{
New Variational Approach in the Theory of Nonequilibrium Stationary Processes
}

$\operatorname{AUTHOR}(S)$ :

Ichiyanagi, Masakazu

\section{CITATION:}

Ichiyanagi, Masakazu. New Variational Approach in the Theory of Nonequilibrium Stationary Processes. 物性研究 1992, 59(1): 49-49

\section{ISSUE DATE:}

1992-10-20

URL:

http://hdl.handle.net/2433/94969

RIGHT: 


\author{
New Variational Approach in the Theory \\ of Nonequilibrium Stationary Processes*) \\ Masakazu Ichiyanagi \\ Nagasaki Institute of Applied Science, Nagasaki, Japan
}

The variation principle by Nakano in the quantum theory of transport processes is reformulated in the form of Hamilton's principle in classical mechanics, which provides us with a new possibility of studying nonequilibrium steady state if we can distinguish the phenomenological (nonlinear) currents from the driving forces. For this purpose the nonequilibrium density matrix is defined for the steady states carrying constant transport currents due to the driving external forces. In terms of this density matrix the generalized response theory is developed to define differential transport coefficients, which satisfy the onsager reciprocity relations. The differential transport coefficients are used to describe the extra dissipation beyond the constant dissipation.

The variation principle developed here is equivalent to the generalized response theory for nonequilibrium steady states. The principle is formulated in terms of the action-like integral

$$
A\left[t_{2} ; t_{1}\right]=\int_{t_{1}}^{t_{2}} d t W[\phi(t) ; \psi(t)]
$$

for any limits $t_{1}$ and $t_{2}$. The stationary value of the functional W[·] equals the Joule heat generated in the system.

*) J. Phys. Soc. Japan 6/('gz) no.7 